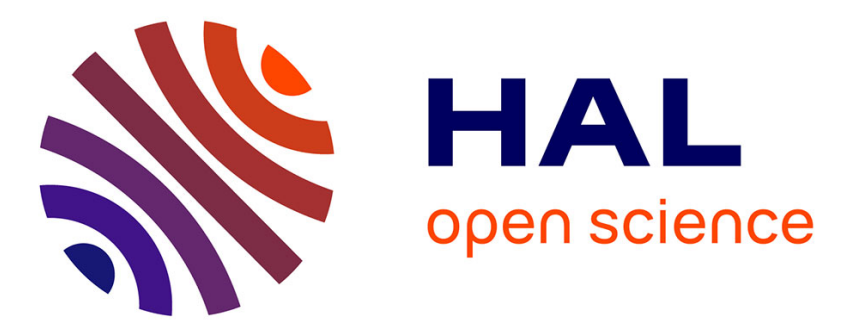

\title{
Corrélation entre les précipitations électroniques et les pulsations du champ magnétique
}

\author{
J.-P. Treilhou, J. Laffont, F. Cambou
}

\section{To cite this version:}

J.-P. Treilhou, J. Laffont, F. Cambou. Corrélation entre les précipitations électroniques et les pulsations du champ magnétique. Revue de Physique Appliquée, 1970, 5 (1), pp.221-226. 10.1051/rphysap:0197000501022100 . jpa-00243364

\section{HAL Id: jpa-00243364 https://hal.science/jpa-00243364}

Submitted on 1 Jan 1970

HAL is a multi-disciplinary open access archive for the deposit and dissemination of scientific research documents, whether they are published or not. The documents may come from teaching and research institutions in France or abroad, or from public or private research centers.
L'archive ouverte pluridisciplinaire HAL, est destinée au dépôt et à la diffusion de documents scientifiques de niveau recherche, publiés ou non, émanant des établissements d'enseignement et de recherche français ou étrangers, des laboratoires publics ou privés. 


\title{
GORRÉLATION ENTRE LES PRÉCIPITATIONS ÉLEGTRONIQUES ET LES PULSATIONS DU GHAMP MAGNÉTIQUE
}

\author{
Par J.-P. TREILHOU, J. LAFFONT et F. CAMBOU, \\ Centre d'Étude Spatiale des Rayonnements, Toulouse (France).
}

\begin{abstract}
Résumé. - Une méthode indirecte du rayonnement auroral s'effectue à l'aide de ballons, permettant ainsi une observation de longue durée. Cependant, pour une meilleure approche de l'origine et du mécanisme des phénomènes auroraux, il est nécessaire d'étudier les corrélations entre les mesures effectuées en ballons et les mesures effectuées au sol (mesures riométriques et du champ magnétique).

L'appareillage au sol utilisé par le Centre d'Étude Spatiale des Rayonnements de Toulouse au cours d'une campagne en Islande en 1967 se composait :

- d'un magnétomètre lent (type variomètre),

- d'un magnétomètre rapide (type fluxmètre),

- d'un riomètre,

et pour l'appareillage embarqué à bord de ballons, d'un spectromètre mis au point au C.E.S.R.

Tout d'abord, deux événements auroraux ont été étudiés en détail (5 août 1967, $00 \mathrm{~h} 00$ 00 h 30 TU ; 8 août 1967, 22 h-23 h TU). Pour ces deux événements, la précipitation électronique

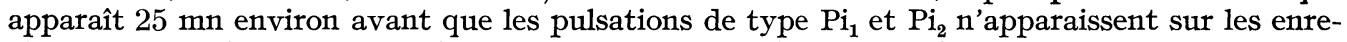
gistreurs magnétiques. L'amplitude des $\mathrm{Pi}_{2}$ et la précipitation électronique passent par un maximum dans le même intervalle de temps.

Une interprétation pourrait être l'apparition d'une instabilité locale du plasma ionosphérique, due à l'arrivée d'un flux croissant d'électrons précipités (provenant de la queue de la magnétosphère), qui engendrerait le courant ionosphérique de type électrojet. Ce courant créerait à son tour d'autres instabilités, expliquant la génération continue des $\mathrm{Pi}_{1}$ et $\mathrm{Pi}_{2}$ pendant toute la phase principale de la baie magnétique.

Il a été fait ensuite une étude de micropulsations magnétiques en perles de 3 à $5 \mathrm{~s}$ observées en présence d'un rayonnement $\mathrm{X}$.

La recherche des périodicités, grâce à une analyse harmonique effectuée selon la méthode de Blackmann et Tuckey, sur des intervalles de temps successifs de $1 \mathrm{mn}$, fait apparaître plusieurs périodes privilégiées $(3,5 ; 4 ; 5,5 \mathrm{~s})$ instables d'une minute à l'autre.

On constate une évolution parallèle de l'amplitude des pulsations magnétiques et du flux d'électrons précipités avec un décalage constant d'environ 5 à $6 \mathrm{mn}$ dans la phase de croissance des deux phénomènes.

Il est alors tentant de conclure que ce sont les émissions hydromagnétiques qui déclenchent la précipitation et la régulent.
\end{abstract}

Introduction. - Une méthode indirecte d'analyse du rayonnement auroral s'effectue à l'aide de ballons, permettant ainsi une observation de longue durée.

Cependant, pour une meilleure approche de l'origine et du mécanisme des phénomènes auroraux, il est nécessaire d'étudier les corrélations entre les mesures faites en ballons et les mesures faites au sol.

Les données ayant servi de base à cette étude ont été obtenues au cours d'une campagne en Islande (1967) organisée par le Centre d'Étude Spatiale des Rayonnements de Toulouse.

L'équipement au sol se composait d'un riomètre et de deux magnétomètres. Un magnétomètre, type variomètre, enregistrait les variations très lentes du champ magnétique terrestre (fréquences comprises entre $10^{-4}$ et $10^{-2} \mathrm{~Hz}$ ). Dans une plage de fréquences comprises entre 0,01 et $1 \mathrm{~Hz}$, les variations du champ magnétique terrestre étaient enregistrées par un système de « barresfluxmètres » mis au point par $M$. Selzer. Le principe de ces « barres-fluxmètres » repose sur deux bobines à noyau de mu-métal, associées à deux galvanomètres fonctionnant en régime suramorti et utilisés comme fluxmètres.

La connaissance précise du spectre en énergie des photons $\mathrm{X}$ à l'altitude du ballon permet de remonter au spectre des électrons primaires et d'en déduire l'évolution en fonction des caractères de la précipitation. Le principe de l'appareillage et les principaux résultats obtenus font l'objet de nombreuses publications (As-67), (As-68), (Ma-67), (Ma-69), (Ba-69), (Co-65), (Co-68), (Tr-69).

L'étude des perturbations magnétiques microscopiques en corrélation avec les précipitations électroniques offre un intérêt considérable. Pour mieux situer la signification des résultats expérimentaux qui suivent, il est intéressant de présenter un système de classification logique des micropulsations magnétiques.

Le tableau I montre la classification et les notations proposées pour les pulsations magnétiques en fonction de leurs propriétés morphologiques. 
TABLEAU I

Type Intervalle De période (s)

\begin{tabular}{lr} 
& \multicolumn{2}{c}{-} \\
& Pulsations continues \\
$\mathrm{Pc}_{1}$ & $0,2-5$ \\
$\mathrm{Pc}_{2}$ & $5-10$ \\
$\mathrm{Pc}_{3}$ & $10-45$ \\
$\mathrm{Pc}_{4}$ & $45-150$ \\
$\mathrm{Pc}_{5}$ & $150-600$
\end{tabular}

PULSATIONS IRRÉGulières

$\begin{array}{lrl}\mathrm{Pi}_{1} & 1 & -40 \\ \mathrm{Pi}_{2} & 40 & -150\end{array}$

I. Apparition simultanée de pulsations de type $\mathbf{P i}_{1}$ et $\mathbf{P i}_{2}$ et d'une précipitation électronique. - Plusieurs auteurs ont déjà souligné l'apparition simultanée des pulsations désordonnées de type $\mathrm{Pi}_{1}$ et $\mathrm{Pi}_{2}$ et d'une précipitation électronique structurée, pendant la phase principale des orages magnétiques. Les périodes décelées dans les flux de particules précipitées couvrent toute la gamme des oscillations hydromagnétiques.

Toutefois, il est bien difficile de dire si ce sont les ondes hydromagnétiques qui causent la précipitation et la modulent, ou si les précipitations de particules engendrent des bruits magnétiques.

La figure 1 représente le début de l'orage magnétique
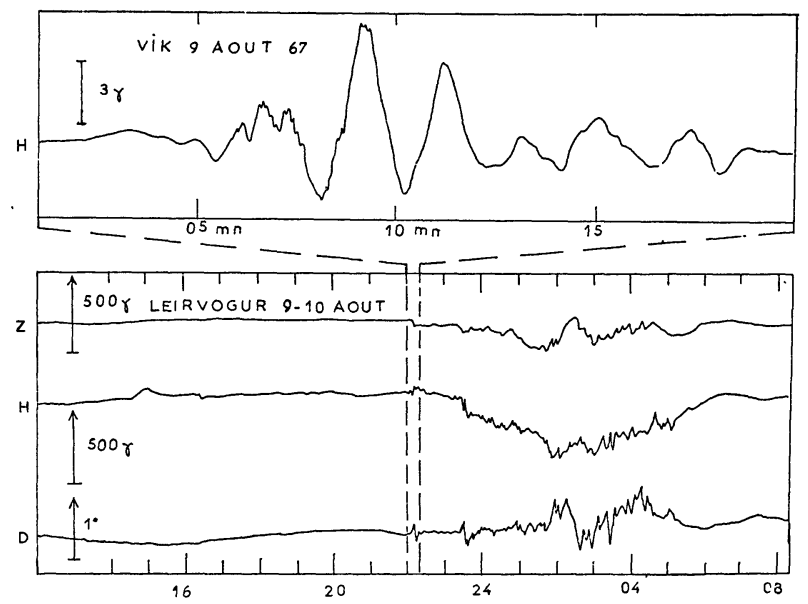

FIG. 1.

survenu le 9 août 1967 en Islande, visible sur le magnétogramme rapide, et sa correspondance sur le magnétogramme lent. Ge train de pulsations amorties qui peut durer de 10 à $15 \mathrm{mn}$ est caractéristique des débuts d'orages magnétiques polaires. Il leur correspond, sur le magnétogramme lent, des variations brutales de pente sur les 3 composantes. Ges caractéristiques ont été retrouvées pour tous les orages magnétiques auroraux enregistrés en Islande entre le 28 juillet et le 15 août 1967. Il est à remarquer qu'il ne s'agit pas de sc ou «sudden commencement » car le dernier en date était intervenu le 11 août 1967, vers 06 h TU.

Ge phénomène n'a pu être retrouvé en zone subaurorale ( $\mathrm{L} 3,7)$, lors d'une campagne d'étude des précipitations aux points conjugués Sogra-Kerguélen (1968). Il semble donc qu'il soit typique uniquement des zones aurorales. La figure 2 illustre un autre exemple de ce
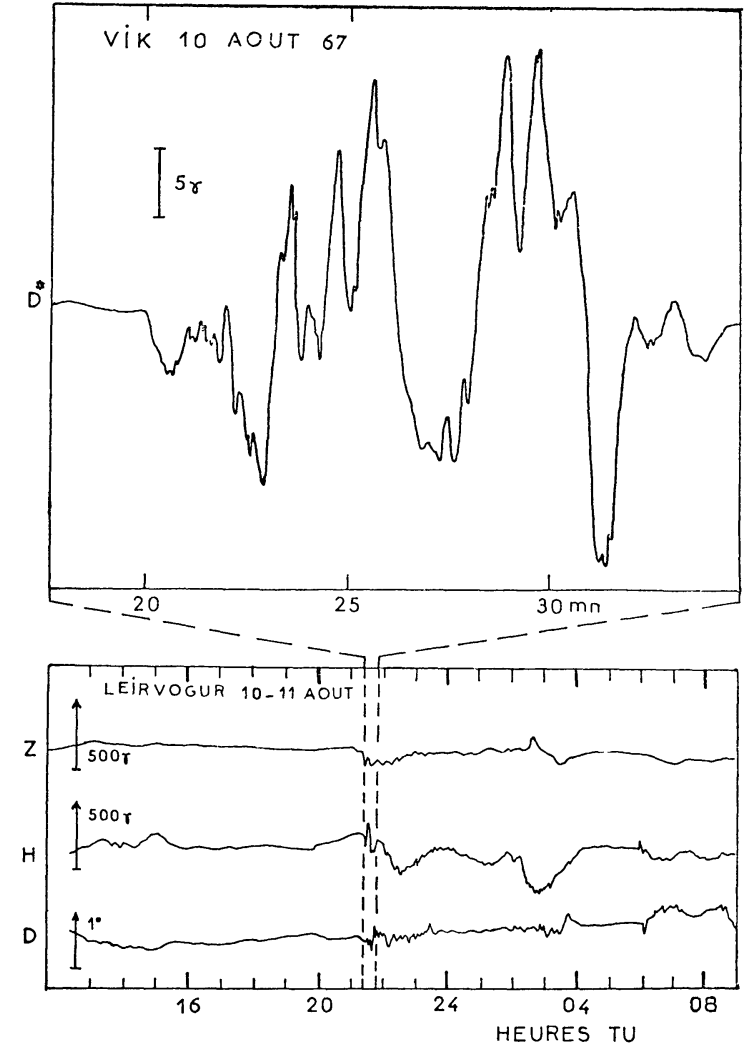

FIG. 2.

phénomène. Les $\mathrm{Pi}_{2}$ de longue période ne présentent plus de caractère amorti, mais sont bien limités dans le temps et il leur est superposé de nombreux $\mathrm{Pi}_{1}$ de fréquences différentes. Le caractère annonciateur d'orage de ces événements les rend très utiles pour une éventuelle décision de lancer de ballons. L'exemple de la figure 1 présente en outre des analogies frappantes avec les $\mathrm{Pi}_{2}$ engendrés par les explosions nucléaires à haute altitude.

Exemple 1 : Événement du 8 août 1967 (22 $h$-23 h TU). - La figure 3 ( $a$ et $b$ ) représente le début d'une baie magnétique survenue le 8 août 1967 et pour laquelle on a disposé des mesures du rayonnement $\mathrm{X}$. Il est intéressant de remarquer que la précipitation électronique au niveau du détecteur apparaît dès $22 \mathrm{~h} \mathrm{TU}$, bien avant les $\mathrm{Pi}_{1}$ et $\mathrm{Pi}_{2}$ (fig. 3 a et b) qui, eux, ne prennent naissance que vers $22 \mathrm{~h} 25 \mathrm{mn}$. L'amplitude des $\mathrm{Pi}_{2}$ croît et atteint son maximum entre $22 \mathrm{~h} 35 \mathrm{mn}$ et $22 \mathrm{~h} 40 \mathrm{mn}$, tandis que la précipitation électronique passe par un maximum dans le même intervalle de temps.

Une interprétation possible pourrait être la suivante. Sous l'effet d'une accélération croissante, il arrive, venant de la queue de la magnétosphère et spiralant le long des lignes de force du champ magnétique terrestre, un flux croissant d'électrons précipités. Lorsque ce flux atteint une valeur suffisante, il apparaît une instabilité locale du plasma ionosphérique, instabilité dont l'effet augmente avec le flux de particules précipitées.

Cette instabilité se déclencherait à partir d'un seuil de particules précipitées et engendrerait le courant ionosphérique de type électrojet. Le plasma ionosphérique, mis en mouvement sous l'effet de l'électrojet, 


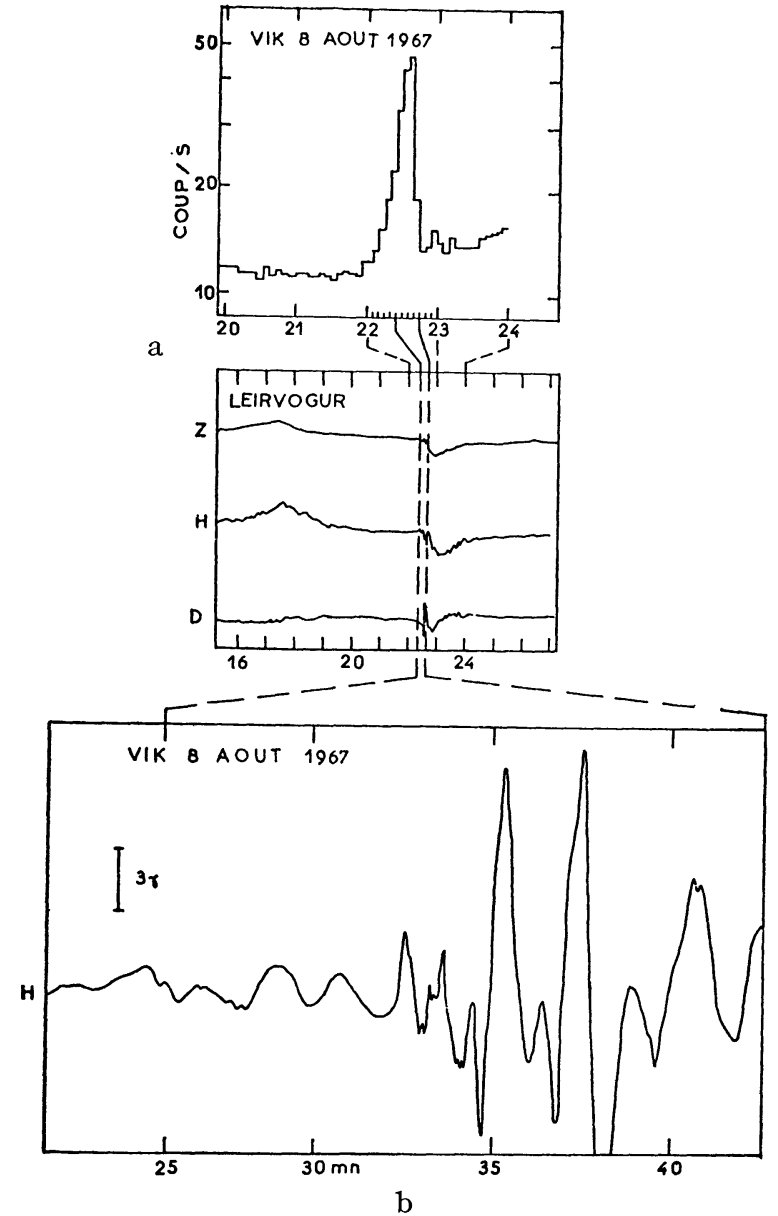

FIG. 3.

créerait à son tour toute une cascade d'autres instabilités, expliquant ainsi la génération continue des $\mathrm{Pi}_{1}$ et $\mathrm{Pi}_{2}$ pendant toute la phase principale de la baie magnétique. L'instabilité initiale aurait pour effet de créer les ondes hydromagnétiques dont les $\mathrm{Pi}_{2}$ enregistrés au sol constituent l'effet visible.

Exemple 2 : Événement du 5 août 1967 (00 $h$ 00$00 \mathrm{~h} 30 \mathrm{mn}$ ). - L'événement magnétique du 5 août 1967 qui est aussi associé à une précipitation électronique est

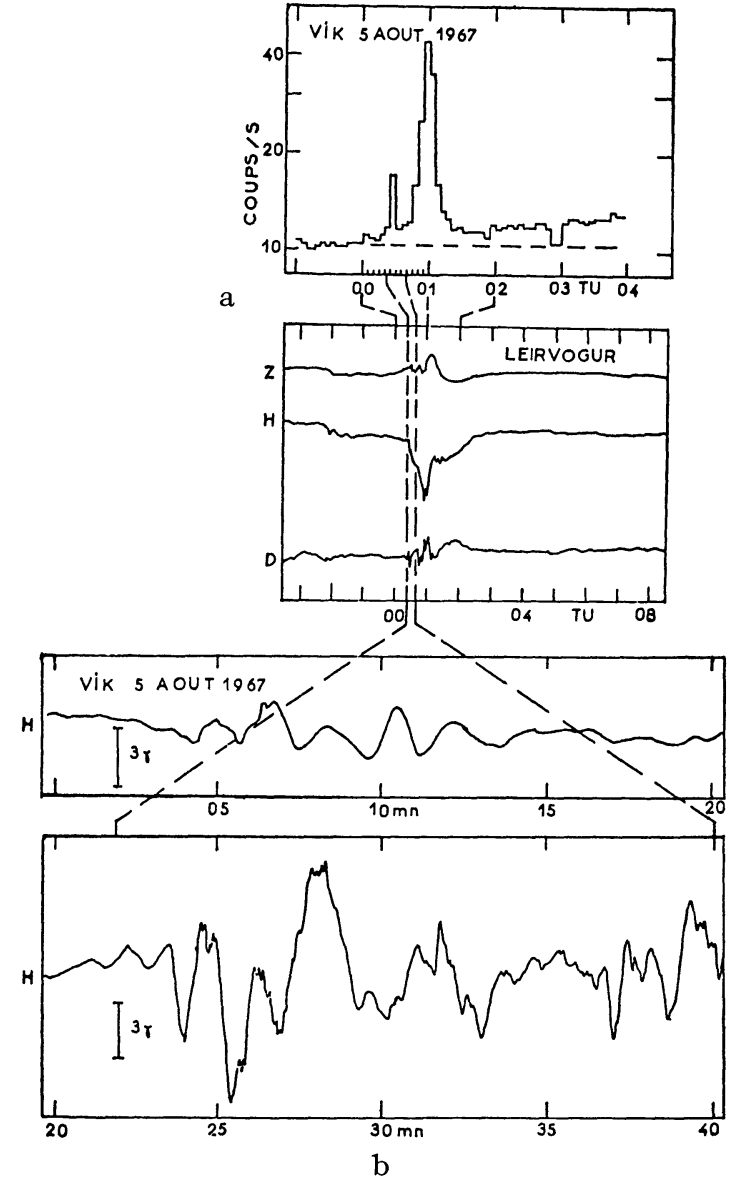

FIG. 4.

représenté sur la figure 4 (a et b). Le magnétogramme rapide montre une série de $\mathrm{Pi}_{2}$ de faible amplitude entre $00 \mathrm{~h} 05 \mathrm{mn}$ et $00 \mathrm{~h} 15 \mathrm{mn}$ TU. Ge type de $\mathrm{Pi}_{2}$ est parfois dénommé de « pré-break-up » $(\mathrm{Pa}-68)$, (Ro-67). Le schéma interprétatif précédent, qui lie l'apparition de l'instabilité à une valeur critique du flux de particules précipitées, reste dans ce cas une hypothèse valable. Le tableau II résume l'évolution du phénomène dans le cadre de l'interprétation suggérée.

TABLEAU II
Heure
MAGnÉtisme

De 00 h $00 \mathrm{mn}$ à 00 h $05 \mathrm{mn}$

De 00 h $05 \mathrm{mn}$ à $00 \mathrm{~h} 15 \mathrm{mn}$

De 00 h 15 mn à 00 h 20 mn

De 00 h $20 \mathrm{mn}$ à $00 \mathrm{~h} 30 \mathrm{mn}$

Vers 00 h $30 \mathrm{mn}$

Calme
Calme

\section{$\mathrm{X}$}

Précipitation de très faible importance qui déclenchera cependant les $\mathrm{Pi}_{2}$ de pré-break-up suivants, mais non une instabilité conséquente

$\mathrm{Pi}_{2}$ de pré-break-up dus à la faible précipitation des $5 \mathrm{mn}$ précédentes

Début et croissance de l'instabilité importante due aux électrons précipités des $5 \mathrm{mn}$ précédentes

L'amplitude des $\mathrm{Pi}_{2}$ de grande période devient maximum
Retour à la valeur du bruit de fond

Début de la précipitation électronique croissante qui sera suffisante pour générer l'instabilité de démarrage de l'électrojet

Groissance du flux d'électrons précipités

Passage du flux des électrons précipités par un maximum 
II. Micropulsations magnétiques en perles de 3 à 5 s. - La figure 5 illustre la forme générale des micropulsations enregistrées par les barres-fluxmètres. On aperçoit le caractère fortement modulé en amplitude de ces pulsations qui permet de les classer dans la gamme des émissions hydromagnétiques en forme de

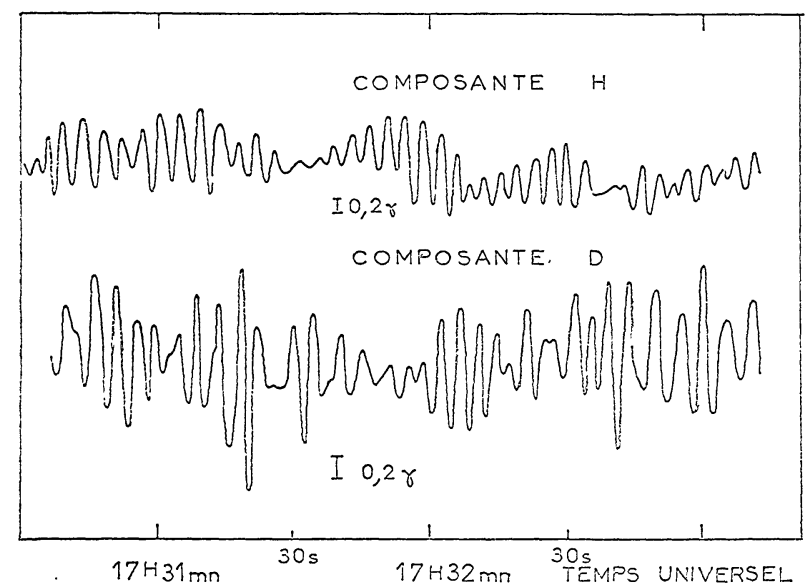

FIG. 5. - Exemple de micropulsations magnétiques en perles, enregistrées le 12 août 1967 à Vik.

perles, ou encore dans la gamme des $\mathrm{Pc}_{1}$ (Camp.-67). Certaines de ces pulsations ont des amplitudes crête à crête qui peuvent atteindre $1 \gamma$. Cette émission hydromagnétique prend naissance à $17 \mathrm{~h} 04 \mathrm{mn}$ TU et se termine à $17 \mathrm{~h} 58 \mathrm{mn}$ TU, alors que la situation magnétique générale est calme $(K p=1)$.

Cet événement se situe donc vers 16 h locales à Vik et la ligne de force dont le pied passe à Vik est nécessairement fermée à cette heure.

L'échantillonnage du signal avec un pas de $0,5 \mathrm{~s}$ a permis la recherche des périodicités grâce à une analyse harmonique effectuée selon la méthode de Blackman et Tuckey (Bl-59). L'analyse sur des intervalles de temps consécutifs de $5 \mathrm{mn}$ (fig. 6) révèle la présence

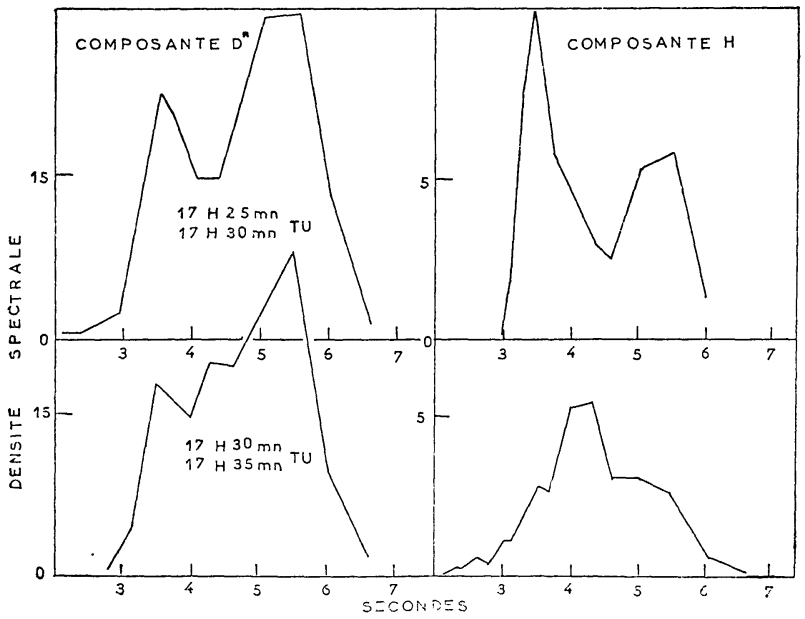

FIG. 6.

Analyse spectrale des oscillations magnétiques.

d'une bande finie de périodes, avec une prédominance des périodes 3,5 et $5,5 \mathrm{~s}$ qui peut cependant évoluer dans le temps. Cette superposition de deux fréquences peut expliquer la modulation générale en amplitude des oscillations par un phénomène de battement.
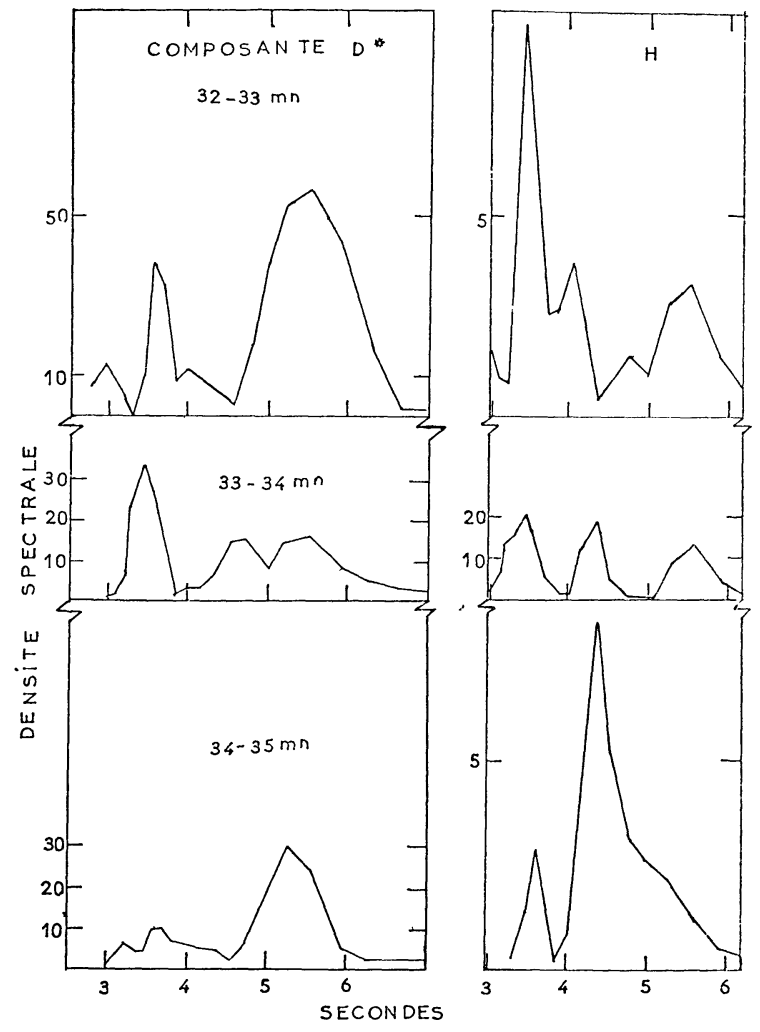

FIG. 7.

La recherche des périodicités sur des intervalles de temps successifs de $1 \mathrm{mn}$ ( fig. 7) fait apparaître plusieurs périodes privilégiées $(3,5 ; 3,75 ; 4 ; 4,25 ; 5,5 \mathrm{~s})$ instables d'une minute à l'autre. Avec cette méthode d'analyse, il est bien difficile de dire s'il y a une légère évolution de chaque fréquence privilégiée en fonction du temps, avec répétition de ces structures évolutives dans le diagramme fréquence-temps, comme c'est le cas pour les « perles » classiques (Ge-62). Toutefois, ce léger glissement de périodes autour de 3,5 et $5,5 \mathrm{~s}$ peut expliquer la modulation d'amplitude désordonnée qui parfois masque l'allure de « perle ».

L'enregistrement du rayonnement $\mathrm{X}$ auroral fait apparaître vers 17 h $10 \mathrm{mn}$ TU une augmentation du taux de comptage dans toute la gamme d'énergie (15-150 keV), soit $6 \mathrm{mn}$ après l'apparition des oscillations magnétiques structurées au sol. La figure 8 montre l'évolution croissante de l'intensité moyennée sur $2 \mathrm{mn}$ du rayonnement $\mathrm{X}$ dans la gamme $15-50 \mathrm{keV}$. Elle atteint un premier maximum ( 3 fois le bruit de fond) à $17 \mathrm{~h} 35 \mathrm{mn}$, se maintient entre $17 \mathrm{~h} 40 \mathrm{mn}$ et $17 \mathrm{~h} 50 \mathrm{mn}$ TU à un niveau proche de sa valeur maximale, puis redécroît lentement pour rejoindre vers $19 \mathrm{~h}$ TU le niveau du bruit de fond.

L'apparition presque simultanée des oscillations magnétiques en « perles » et d'une précipitation électronique a conduit tout naturellement à l'analyse du caractère évolutif des deux phénomènes.

Après agrandissement de l'enregistrement graphique, le recensement des amplitudes des oscillations magnétiques a été effectué dans des intervalles de $0,1 \gamma$. La précision sur la détermination des amplitudes est estimée à $\pm 0,04 \gamma$. La figure 9 montre les histogrammes des amplitudes des " perles » sur des intervalles de $5 \mathrm{mn}$ ainsi que l'évolution correspondante de l'intensité du rayonnement $\mathrm{X}$ moyennée sur $1 \mathrm{mn}$. L'évolution 


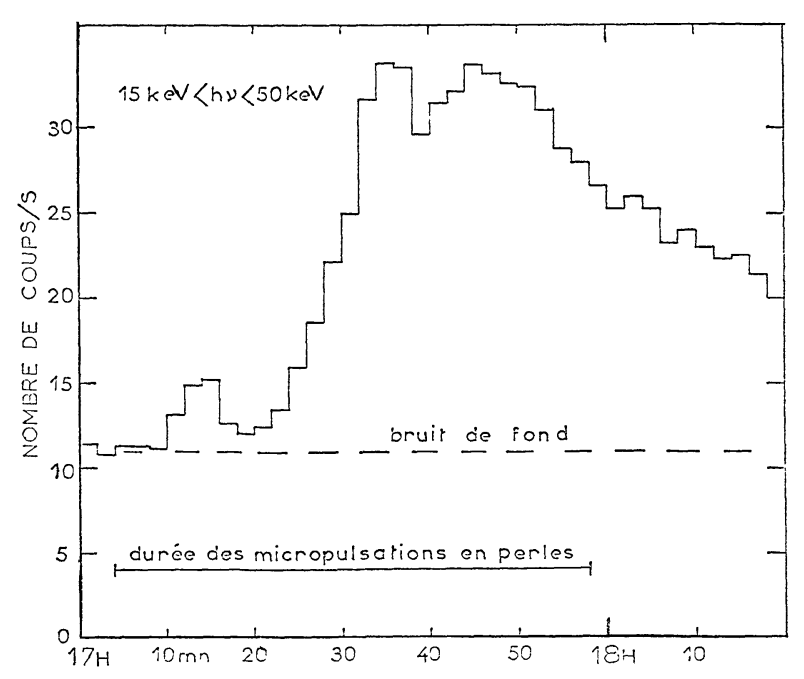

Fig. 8. - Flux de rayons $\mathrm{X}$ intégré sur $2 \mathrm{mn}$ dans la gamme $15-50 \mathrm{keV}$.

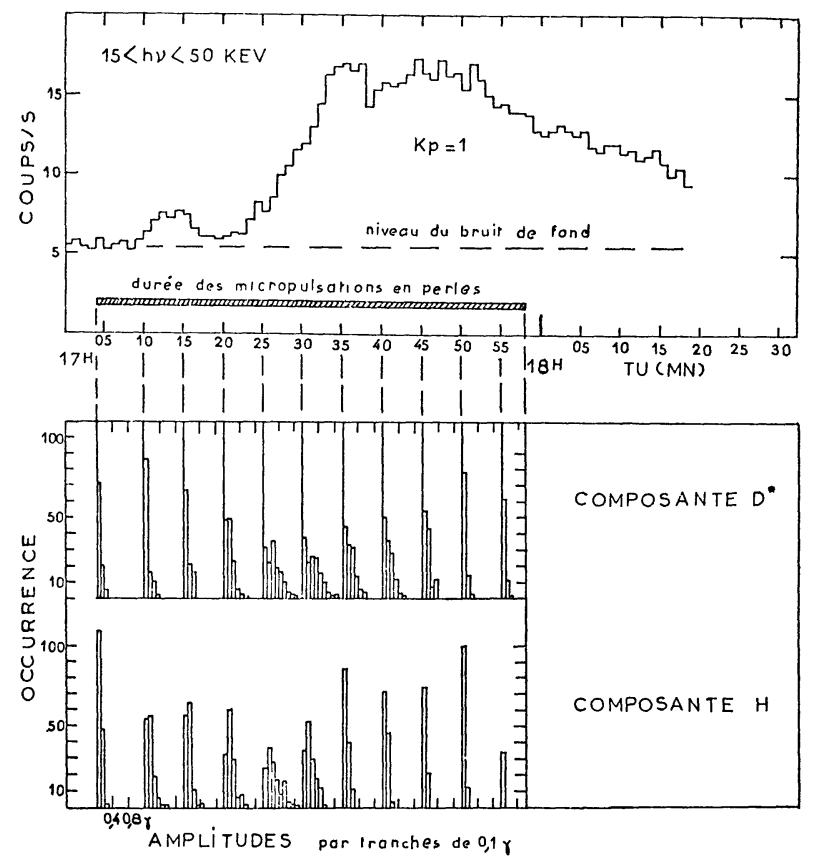

FIG. 9.

des histogrammes des amplitudes au cours du temps montre que la proportion des grandes amplitudes des oscillations magnétiques augmente régulièrement pour atteindre un maximum sur les deux composantes vers $17 \mathrm{~h} 30 \mathrm{mn}$, soit environ $5 \mathrm{mn}$ avant que la précipitation électronique ait atteint son maximum. On constate donc une évolution parallèle de l'amplitude des pulsations magnétiques et du flux des électrons précipités avec un décalage constant d'environ 5 à $6 \mathrm{mn}$ dans la phase de croissance des deux phénomènes.

L'interprétation du déphasage de $6 \mathrm{mn}$ entre les deux phénomènes est délicate. Si l'on admet que l'apparition des grandes amplitudes est commandée uniquement par la source émettrice des oscillations, et non par des modifications du coefficient de transmission de l'ionosphère, la puissance véhiculée par le signal hydromagnétique sur sa trajectoire va croître et passer par un maximum.

L'énergie cédée à l'ionosphère subira la même évolution et pourra se transférer au même rythme aux particules. Si ce sont des électrons piégés qui précipitent sous l'effet de l'apport d'énergie dû aux ondes, plus cette énergie sera grande, plus la quantité d'électrons précipités sera importante.

L'influence des précipitations de particules sur l'apparition des oscillations en perles, quelquefois mentionnée (Wi-66), est généralement controversée (Camp. et al.-65, Mc et al.-65). L'exemple original du 12 août 1967, de précipitation de particules liée à des oscillations en perles, traduirait au contraire une influence des oscillations magnétiques en perles sur la précipitation électronique.

Conclusion. - L'étude conjointe des pulsations magnétiques irrégulières et du flux de rayons $\mathrm{X}$ a conduit à une interprétation nouvelle des débuts d'orages polaires modérés, grâce aux déphasages mis en évidence. Dans le domaine des oscillations magnétiques structurées en perles dans la gamme 3-5 s, une évolution parallèle de l'amplitude des micropulsations magnétiques et d'un flux modéré de rayons $\mathrm{X}$ avec un déphasage d'environ $6 \mathrm{mn}$ a pu être dégagée. Cette évolution parallèle conduit à imaginer un transfert de puissance des oscillations structurées à une certaine classe de particules piégées.

Il importe maintenant de confirmer qu'il ne s'agit pas de phénomènes aléatoires, ce qui nécessite l'exploration continue de la magnétosphère par satellites, des mesures simultanées à grande échelle au sol et à bord de ballons en zone aurorale.

\section{BIBLIOGRAPHIE}

Astor (J. L.) et Co'TIN (F.), Description d'un dispositif de codage destiné à la spectrométrie (utilisation des ballons pour la recherche scientifique, Colloque international C.N.E.S.), 1967.

ASTOR (J. L.), Réalisation d'un dispositif de codage et de décodage. Application à la spectrométrie fine du rayonnement $\mathrm{X}$ auroral, Thèse de Spécialité, Toulouse, 1968.

BARRIa (D.), Mécanismes et spectrométrie des précipitations électroniques dans les zones aurorales, Thèse de Spécialité, Toulouse, 1969.

BLACKMAN et TUkEX, The measurements of power spectra, Dover publications, New York, 1959.
Cambou (F.), Cotin (F.) et Reme (H.), Mesure du rayonnement X auroral, Space research, 1966, 7, NorthHolland, Publishing Company, 635.

Cambou (F.), Maral (G.) et Treillhou (J. P.), Résultats récents sur l'étude du rayonnement $\mathrm{X}$ auroral. Communication G3 présentée au XIe Congrès du Cospar, Tokyo, 1968.

Campbeil (W. H.) et Matsushita (S.), Auroral zone geomagnetic micropulsations with period of 5 to $30 \mathrm{~s}$, J.G.R., 1962, 67, 555-573.

Campbeil (W. H.) et Matsushita (S.), Physics of Geomagnetic Phenomena, II, Academic Press, 1967, 823.

Chapman (S.), Geophysics : The Earth Environment, édité par DE WITT, HIEBLOT, LEBEAU, Les Houches, 1962. 
CoTin (F.), Thèse de Docteur-Ingénieur, Toulouse, 1965.

Cotin (F.) et MARAL (G.), Stabilités des ballons stratosphériques. Note technique présentée au C.N.E.S., Paris, 1968.

GENDRIN (R.) et Stefant (R.), Analyse de fréquence des oscillations en perles, C. R. Acad. Sci., 1962, 255, 752 .

GENDRIN (R.), E.B.F. et micropulsations, Annales de Géophysique, 1967, 23, 159.

GENDRIN (R.), LACOURLY (S.), SHePeTNOV (R. V.) et Troitskaya (V. A.), Caractéristiques des pulsations irrégulières de période décroissante (I.P.D.P.) et leurs relations avec les variations de flux des particules piégées, Planetary and space science, 1967, 15, 8, 1239.

GENDRIN (R.), KAZAK (B.), LAURENT (G.) et RASPOPOV (O.), Caractéristiques nouvelles de certaines oscillations hydromagnétiques irrégulières, C. R. Acad. Sci. Paris, 1966, 263, 841.

HEIRTZLER (J. R.), Regular oscillations with periods of $5 \mathrm{~s}$ to $7 \mathrm{mn}$ : the experimental approach, Rep. no 8815 NBS, Boulder, 1965, Colorado.

MARAL, (G.), Spectromètre directif destiné à l'étude $\mathrm{du}$ rayonnement $\mathrm{X}$ auroral (utilisation des ballons pour la recherche scientifique, Colloque international C.N.E.S.), 1967.

MARAL (G.), Thèse de spécialité, Toulouse, 1967.

MARAL (G.), Étude du rayonnement $X$ lié aux précipitations d'électrons. Exposé présenté à la IXe Réunion du Sparmo, Toulouse, 1967.

MANeuvrier (J.) et MARAL (G.), Configuration et déplacement des précipitations nocturnes d'électrons auroraux, C. R. Acad. Sci. Paris, 1969, 268.

Maneuvrier (J.), Thèse de $3^{\text {e }}$ cycle, Toulouse, 1969. MCPherron (R. I.) et WARD (S. H.), Pearl pulsations interplanetary magnetic field sector correlation. Communication présentée à "URSI Spring Meeting ", Washington, avril 1966.
PARKs (G. K.), MCPherron (R. L.) et Coroniti (F. V.), Relation of energetic electron precipitation and geomagnetic micropulsation to auroral substorms, Ann. Geophys., 1968, 24, 621.

Selzer (E.), C. R. Acad. Sci. Paris, 1956, 242, 1422-1425.

TreillHou (J. P.) et LAFFONT (J.), Micropulsations magnétiques en perles de 3 à $5 \mathrm{~s}$ observées en présence d'un rayonnement X, Ann. Géophys., 1969, 25, 647.

Treillhou (J. P.), Thèse de $3^{\mathrm{e}}$ cycle, Toulouse, 1969.

Troitskaya (V. A.), Pulsations of the earth's electromagnetic field and their connection with phenomena in the high atmosphere, J.G.R., 1961, 66, 5-18.

Ullaland (S. L.), Trefali (H.), KRemser (G.) et BEWERSDORFF (A.), Observations of large scale coherent pulsating electron precipitation events in the auroral zone, accompanied by continuous geomagnetic pulsations. Communication présentée au Colloque Inter-Unions sur les relations Soleil-Terre, Belgrade, 1966.

VICTOR (L. J.), Correlated auroral and geomagnetic micropulsations in the period 5 to $40 \mathrm{~s}$, J.G.R., 1965, 70, 3123.

VOELKER (H.), On the latitude dependance of Pc-type micropulsations, Results of observations on a northsouth profile through Europe, Rep. no 8815 NBS, Boulder, Colorado, 1965.

WILHEIM (K.), Geomagnetic micropulsations in the northern auroral zone compared with X-ray measurements. Communication présentée au Colloque Inter-Unions sur les relations Soleil-Terre, Belgrade, août 1966.

WRIGHT (Sir C. S.) et LoKkEN (J. E.), Geomagnetic micropulsations in the auroral zones, Canad. J. Physique, 1965, 43, 1373. 